\section{Predicting that comet Halley is dark}

SiR-As I write this letter in early March only the scantiest information has come out of the Giotto mission. One of the most interesting preliminary findings was that comet Halley appeared to be quite dark. This may seem to be inconsistent with the high degree of reflectivity one might expect from an icy comet nucleus ${ }^{1}$, implying that something extra is required to blacken the surface. I should like to show that a very low reflectivity is a predicted ${ }^{2}$ and natural consequence of the comet nucleus being a fluffy aggregate of small solid intersteller particles ${ }^{2}$ each of which is largely water ice and at least moderately reflecting. The reflectivity of the comet is low because it is an open structure and light which penetrates it is internally absorbed by being scattered many times by the individual small particles which are its components.

The first stage of interstellar particle coagulation in the presolar nebula which is assumed to lead ultimately to the comet nucleus is shown schematically in Fig. 1 by a model containing 91 individual particles. Each particle is a mean representative of a size distribution of interstellar grains which have fully accreted all the condensable molecules from the dense molecular cloud out of which the solar system was born. It consists of a silicate core, an inner mantle of complex organic refractory material which has evolved by ultraviolet photoprocessing over hundreds of millions of years before comet coagulation, and an outer mantle of volatile ices in which $\mathrm{H}_{2} \mathrm{O}$ is dominant ${ }^{3}$ and which contains $\mathrm{S}_{2}$ molecules which could only have been produced in the interstellar dust before coagulation ${ }^{4}$. These particles are typically $\sim 0.5 \mu \mathrm{m}$ thick and at least three times as long 5 . Imbedded in the outer mantle are many small $(\sim 0.01 \mu \mathrm{m}$ radius) particles (some strongly absorbing) which show up in space as blocking the ultraviolet radiation of stars ${ }^{6}$

The porosity shown in Fig. 1 was derived by comparing the density of comet dust with the mean density of meteors which are known to be comet debris. Although the mean density of the nonvolatile silicate core-organic refractory mantle interstellar grains (no outer icy mantle) is about $1.9 \mathrm{~g} \mathrm{~cm}^{-3}$, a characterisitic mean meteor density of $0.2 \mathrm{~g} \mathrm{~cm}^{-3}$ is observed $^{7}$. This obviously implies a high degree of porosity. The organic refractory component is the largest part of the comet dust after the volatiles evaporate. Taking into account the volume of volatile ices in the precometary dust (and thus in the original comet matter) one arrives ${ }^{2}$ at a mean packing factor for the comet of no more than about 0.4 (60\% empty space). This is what has been pictured for the 4 $\mu \mathrm{m}$ aggregate in Fig. 1. An even fluffier comet nucleus structure is probable since the meteor particles must undergo some collapse after evaporation of the volatile components ${ }^{8}$.

It is therefore reasonable to approximate the light scattering by a comet as if it were a cloud of small particles each of which is characterized by an albedo $\alpha$ and a scattering asymmetry factor $g$. The individual particle albedo is defined as the ratio of the light scattered by the particle to the light it both absorbs and scatters (no absorption: $\alpha=1$; total absorption: $\alpha=$ 0 ). The asymmetry factor, defined as the mean value of the cosine of the scattering angle, is essentially the difference between the forward scattered and the backward scattered light (spherically symmetric scattering: $g=0$; no back scattering: $g$ $=1$ ). At visual wavelengths the normal interstellar grains (no icy coating) have an albedo $\alpha=0.6 \pm 0.1$ and an asymmetry factor $g=0.8 \pm 0.1$ (ref. 9). It is a well known property of the scattering of light by small particles that in the size to wavelength range of interstellar dust, a further increase of particle size by the addition of icy mantles leads to an increase in the asymmetry factor up to $g=0.9$ (ref. 10 ). The very strongly forward directed scattering implies that light impinging on a particle at the comet surface mostly continues inward where it may be absorbed by repeated scatterings within the body.

Let the forward and backward scattering fractions be $F$ and $B$ so that $F+B=1$. Using $g=F-B$ the backward scattered fraction is $B=(1-g) / 2$. Since only a part, $\alpha$, of the incident radiation per particle is scattered and, since we shall assume that all the forward scattered radiation is lost, the net back scattering by the sum of the individual particles filling the surface of the comet is the total reflected light. This gives a reflectivity of $A=\alpha(1-g) / 2$. So

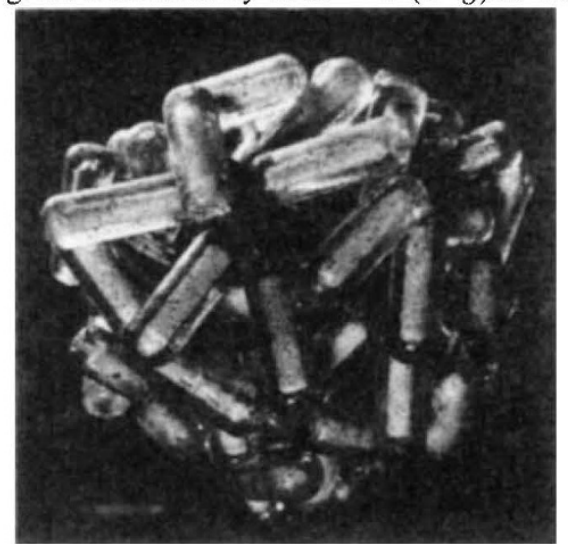

Fig. 1 A model of randomly coagulated coremantle dust grains. Shown is an ensemble of 91 particles incorporating within their outer icy mantles the very small particles of the interstellar dust population accreted in the final stages of condensation. The degree of volumetric packing is $0.4(60 \%$ open space $)$ and the overall dimension is scaled to about $4 \mu \mathrm{m}$. for $\alpha=0.6$ and $g=0.9$ only about $3 \%$ of the light is reflected. This simple but physically reasonable approximation for the reflectivity matches remarkably closely the value $A=0.028$ obtained from very accurate calculations of Van De Hulst ${ }^{11}$.

For the same reason, large comet dust particles should similarly exhibit low reflectivities, as may have been detected in comet Crommelin ${ }^{12}$. Judging by the high infrared emissivity of interplanetary particles well beyond the Earth's orbit (S.S. Hong, personal communication) they too, as young comet debris, are highly absorbing because of their fluffy character.

Other critical predictions of this model of the nucleus are: (1) A preponderance of the organics $\mathrm{C}, \mathrm{O}, \mathrm{N}$ relative to the rocky elements $\mathrm{Si}, \mathrm{Mg}, \mathrm{Fe}$ by a factor of five or greater in comet dust grains because of the initially high ratio of the organic refractory to silicate volume in the interstellar core-mantle grains ${ }^{2}$. (2) Individual coremantle particles of order $0.15 \mu \mathrm{m}$ radius and mass about $3 \times 10^{-14} \mathrm{~g}$ resulting from aggregate breakup. (3) A very large number of very small $(\sim 0.01 \mu \mathrm{m})$ particles of mass of about $2 \times 10^{-17} \mathrm{~g}$ released from the evaporating icy material ${ }^{13}$. (4) A very low comet heat conductivity leading to high surface heating and a concomittant high vaporization rate due to the very porous surface area.

Should the Giotto and Vega missions confirm these predictions we may well be able to say that comets provide a direct observation of the interstellar dust as it existed $4.5 \times 10^{9}$ years ago and that a comet-nucleus-sample-return mission will accomplish what no amount of remote sensing of the interstellar medium will ever make possible.

Laboratory of Astrophysics,

University of Leiden,

Wassenaaresweg 78, Postbus 9504,

2300 RA Leiden, Netherlands

Whipple, F. in Cosmic Dust (ed. McDonnell, J.A.M.) 1-73 (Wiley, New York, 1978).

2. Greenberg. J.M. in Comets (ed. Wilkening, L.L.) 131-163 (University of Arizona Press, Tucson, 1982); in Asteroids, Comets, Meteors Vol. II (eds Lagerkvist, C.-I., Lindblad, B.A., Lundstedt, H. \& Rickman, H.) 221-223 (Uppsala University Press, 1986)

Greenberg. J.M. in Submillimetre Astronomy (eds Beck man. J.E. \& Phillips, J.P.) 261-306 (Cambridge University Press. 1982)

4. A'Hearn, M.F. \& Feldman, P.D. in Ices in the Solar System (eds Klinger, J., Benest, D., Dollfus, A. \& Smoluchowski, R.) 463-471 (Kluwer, Dordrecht. 1985)

5. Greenberg, J.M. in Stars and Stellar Systems Vol. VII (eds Middlehurst, B.M. \& Aller, L.H.) 221-364 (University of Chicago, 1968).

6. Greenberg, J.M. \& Chlewicki, G. Astrophys. J. 272, 563-578 (1983)

Verniani, F Space Sci, Rev, 10, 230-261 (Reidel, Dordrecht, 1969)

8. Mukai, T. \& Fechtig, H. Plant. Space Sci. 31, 655-658 (1983).

9. Savage, B.D. \& Mathis, J.S. A. Rev. Astron. Astrophys 17, 73-111 (1979)

10. Martin, P.G. Cosmic Dust (Clarendon, Oxford, 1978).

11. Van de Hulst, H.C. Multiple Light Scattering Vol. 2, 369 (1985).

12. Hanner, M.S., Knacke, R., Sekanina, Z. \& Tokunaga, A.T. Astron. Astrophys. 152, 177-181 (1985)

13. Greenberg, J.M. Adv. Space Res. 4, 211-212 (1984). 